\title{
Sustained Knowledge Management by Organizational Culture
}

\author{
Birgit Lemken, Helge Kahler and Markus Rittenbruch \\ Institute of Computer Science III \\ University of Bonn \\ Roemerstr. 164 \\ D-53117 Bonn, Germany \\ \{lemken|kahler|mr\}@cs.uni-bonn.de
}

\begin{abstract}
Preserving and fostering knowledge is the vital interest of a network-like virtual organization. The decentralized and geographically distributed organizational structure inhibits knowledge flow. The particular conditions of knowledge management in virtual organizations are discussed by the example of an empirical study we carried out in a service company. We analyze how technical and organizational aspects influence knowledge sharing and transfer. Particular emphasis is placed on supporting knowledge transfer. It is shown how an existing knowledge sharing tradition had to be extended and which drawback it had concerning technology use. To improve knowledge flow within the organization a web-based knowledge base was introduced. This initiated a process of externalizing tacit knowledge and is meant to introduce new ways of knowledge transfer to the organizational culture. In fluid virtual organizations sustained knowledge management means to establish an organizational memory that is flexible and adaptive to changing requirements. This is best achieved by a strong organizational culture that emphasizes knowledge sharing by the use of various communication channels.
\end{abstract}

\section{Introduction}

One of the key assets of enterprises is their knowledge. Effective use of internal knowledge creates advantages for market competition. What is meant by knowledge? The way to an answer leads through the history of eastern and western philosophy [1]. Certainly, knowledge is based upon information and bound to people [2]. We understand knowledge as actively processed information and personal experience, being aware that we often talk about knowledge when information is meant. As knowledge is not static but highly dynamic, the question is how to establish a flow of information and know-how. In classical organi- zations this is partly realized by hierarchical structures, where knowledge is connected to hierarchical levels. In virtual organizations knowledge processing is inhibited by decentralized structures and geographical distribution. In this article we will investigate issues concerning sustained knowledge management in virtual organizations. Using the example of a case study we carried out in a teamoriented virtual organization we will analyze how technical and organizational aspects influence knowledge processes. Particular emphasis will be placed on the question of how knowledge visibility and transfer can be supported. A key to sustained knowledge management is organizational culture that forms the environment in which information and know-how can flow.

First we give a short overview of virtual organizations and the special case of team-based ones. After outlining some issues of knowledge management in the context of virtual organizations we present our case study Sigma, a team-based freelancer organization. The study was carried out on behalf of the projects VIRTO ${ }^{1}$ and InKoNetz ${ }^{2}$. A discussion of our knowledge related findings concludes the article.

\section{Virtual organizations}

In recent years, new concepts of organizations have experienced an enormous upswing, among them the virtual organization [3,4] and the fractal organization [5]. A common aspect of most of these organizational forms is that they are partially structured as networks.

A whole group of organizational forms is classified as virtual organizations, e.g. when small enterprises organize work along the entire value chain (Walden Paddlers [6]). In addition these forms of enterprises are not restricted to one country, e.g. Rosenbluth International Alliance is a

1 This work was funded by MSWWF, NRW, Germany.

2 This work was funded by EU Initiative ADAPT. 
global cooperative alliance of independent travel agencies [7].

Two different sources have created the term virtual organization. Davidow and Malone coined the phrase in the book "The Virtual Corporation" [3]. They stress the temporal aspect: a corporation forms to realize a business goal and separates after achieving it. During the eighties Mowshowitz developed a model of the virtual organization where task requirements and satisfiers are strictly separated. At the very moment a request is received the satisfiers are assigned to the task [8]. Anyway, some advantages of virtual organizations are improved resource utilization and greater flexibility at lower costs.

Though a generally approved formal definition of the virtual organization is not at hand, the common understanding covers the cooperation of legally independent partners which contribute their core competencies to a vertical or horizontal integration and appear as one organization to the customer. The organization is often established for a limited time and participants are usually geographically distributed. Compared to classic enterprises they are less rigidly structured. Decentralized organizations with almost no hierarchies rely heavily on communication and coordination of internal processes. Therefore, one of the prerequisites for virtual organizations is the existence of a technical infrastructure to support communication and collaboration [9]. In an ideal virtual organization documents are kept electronically, communication takes place computer-mediated, and clear external boundaries of the organization are difficult to establish [10]. A good overview of the different definitions and how virtual organizations relate to networks, joint ventures, strategic alliances, agile enterprises, valueadding partnerships or clan organizations is provided in [4].

\subsection{The team-based virtual organization}

A special variety of virtual organization is constituted by cooperation of freelancers or very small enterprises. Such an organization bundles the capabilities of its members and sells them as services. The hierarchical structure of a virtual organization is mainly horizontal in contrast to multi-level hierarchies in traditional enterprises. Its structure is best characterized as a polycentric network where project teams form temporary sub-structures. Local centers may change or remain static.

There are hardly any permanent organizational functions bound to people. A project leader will only manage her own project. Work that relates to the whole organization is done in projects within the enterprise or given to an external service provider. The ability to form a project team that perfectly meets the project task is considered as one of the big advantages of a virtual organization that makes it ready for competition. Jarvenpaa and Shaw write "The basic building block of virtual organizations is a virtual team" [11]. It is the center of activity in the organization.

The customer perceives the organization as a whole not regarding its virtual characteristics. By combining capabilities, know-how and qualities of diverse sources it is possible to cover a broad market segment. Each member profits from this. Acquiring projects, managing customer care and organizational issues as accounting or marketing is a task for the virtual organization as a whole. Viewed from inside, the enterprise consists of several project teams that are highly diverse with regard to magnitude, number of members or duration.

While the project teams only form for a limited time the virtual organization as a whole heads for permanent existence. Therefore, it is crucial to save and procure knowledge and know-how that was acquired in particular projects. A virtual organization depends on the commitment of each of its members. The relationship of members to the enterprise and to each other is crucial. Quality of work and motivation are directly connected to the depth of ones personal commitment. Due to their geographically distributed locations members work separately and isolated. Developing a community is important though inhibited by distribution and mediated communication. Some enabling factors for team-based virtual organizations have been identified (cf. [9]):

- Lacking rigid formal obligations, trust among the partners of a virtual organization is considered as a vital requirement for collaboration.

- The ability to build flexible teams is highly important to ensure the organization's flexibility to react to market demands.

- As the virtual organization is less structured than other organizations communication and cooperation procedures require special organizational and technical support .

As the support of cooperation and coordination is a general demand we will not consider it in detail. For an explanation of basic concepts cf. [12].

\subsection{Knowledge management in virtual organi- zations}

A virtual enterprise can be regarded as a typical knowledge organization. "It is the most radical form to realize the customer- or task-oriented integration of information and knowledge within a temporal and fluid configuration" [13]. While classic enterprises organize parts of their knowledge along organizational structures and hierarchies, virtual enterprises lack this option. On the other side they depend heavily on effective knowledge management: 
- they are competitive by bundling capabilities and competencies, i.e. they sell their know-how,

- the knowledge resources of participants are unknown at the start of collaboration,

- people leave the enterprise and take their knowledge with them,

- recently acquired know-how is difficult to preserve at the end of a collaboration,

- different viewpoints and requirements of partners make it difficult to represent knowledge.

Knowledge management in virtual organizations is difficult for the following reasons:

- work is carried out under high time pressure,

- the dynamic and fluid organizational structure inhibits establishing an infrastructure for knowledge sharing,

- for each member knowledge sharing is coupled with the risk of losing competitive advantages,

- temporally limited cooperation leads to knowledge lost at the end of a work relation.

The formation of a virtual organization can be regarded as a bundling of competencies and know-how to act on the market. Coupling knowledge resources is quite demanding for the participants as it requires technical and organizational collaboration. To cooperate the partners must share their knowledge while at the same time they have to ensure their own business activities. To overcome the tension between competition and cooperation trust plays an important role as it is a base of social cohesion [11]. Trust constitutes a relation ready for open and risky information exchange. It is prerequisite and consequence of successful business relations. The development of trust is restricted by mediated communication and temporal work relationships. Lipnack and Stamps regard trust as the believe in integrity, fairness and reliability of a person or organization based on past experience [14]. At least trust between people can only be established by personal contacts and needs time to grow.

Seen from a social view, cohesion, i.e. forces that attract members to common goals, establish identity and shared values, is a central factor to make a virtual corporation work. Organizational culture is defined as patterns of shared values and beliefs that over time produce behavioral norms adopted in solving problems [15]. Similarly, Schein notes that culture is a body of solutions to problems that have worked consistently and are taught to new members as the correct way to perceive, think about, and feel in relation to those problems [16]. The sum of these shared philosophies, assumptions, values, expectations, attitudes, and norms bind the organization together. Moreover, the regulation of organizational processes works better by means of collective values and models than by means of formal structure [17]. Organizational culture, therefore, may be thought of as the manner in which an organization solves problems to achieve its specific goals and to maintain itself over time. Moreover, it is holistic, historically determined, socially constructed and difficult to change [15]. Organizational culture is a key to collaboration in virtual organizations.

The role of knowledge management in this context of team-oriented virtual organizations is

- to identify, open up and gather sources of information and knowledge,

- to organize these sources and resources and to make them available,

- to support knowledge acquisition and development as well as transfer and provision.

As stated in [1] knowledge appears in two forms: tacit knowledge that is subjective and difficult to transmit, and explicit knowledge that is objective and easy to communicate. Knowledge can be transformed between these two forms, e.g. learning transforms explicit knowledge into tacit. There are a lot of partial technical solutions to knowledge management such as document management systems, electronic mail management [18] or work flow management systems (process-oriented knowledge). A more holistic view is taken by Stein and Zwass by introducing the Organizational Memory Information System (OMIS) [19]. It integrates past and present knowledge by making it explicit for future use.

The idea behind an "organizational memory" (cf. [20, $21,22]$ ) is to cover several forms of knowledge (Figure 1). As tacit knowledge is difficult to externalize, expertise management instead of managing facts is growing in importance [23]. However, virtual organizations have fuzzy boundaries and suffer from the fluctuation of members which limits the option of expertise management.

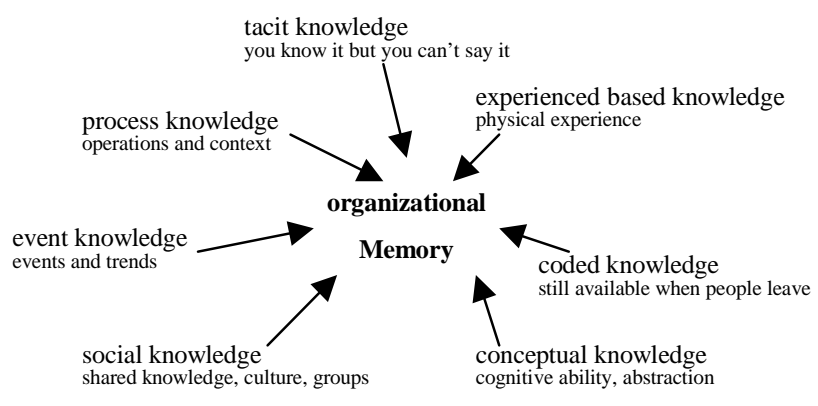

Figure 1. Knowledge types

\section{Case study: Sigma}

The particular situation of knowledge management in team-oriented virtual organizations was studied by empirical work, carried out over the last three years in a German service company. We describe the development 
and structure of this organization, its technical support for information and communication and how it changed over time. Communication processes and knowledge transfer are analyzed with respect to organizational culture.

\subsection{Methodology}

To gain an understanding of the organization as a whole and the viewpoints of the individual members we used several empirical methods. We participated in an internal working group called "Sigma information and knowledge management". This group meets about once a month. We analyzed the use of SigSys, a bulletin board system introduced in 1996. As participating users we had access to the system and its discussion groups. We participated also in a working group on organizational culture set up by Sigma. Finally semi-structured interviews were carried out. Using a set of predefined questions we conducted sixteen narrative, face-to-face interviews and fourteen telephone interviews with members of Sigma, lasting between twenty and sixty minutes. The interviewees represented several functions (management, project managers, project members) and were distributed all over Germany. While participating in working groups and observing the use of SigSys gave us insight into organizational matters the interviews clearly demonstrated how heterogeneous personal views were.

\subsection{Sigma's history}

Sigma was founded seven years ago as a freelancer network offering training courses and consulting services. In the beginning they were about 20 members knowing each other through former collaboration. During the last years Sigma underwent a rapid growth. Today about 200 consultants and trainers with home offices in various parts of Germany are members of Sigma. The rapid growth led to changing organizational requirements, especially with regard to information distribution and communication. By introducing a bulletin board system (SigSys) computer mediated communication took up a role in communication and information sharing. By establishing eleven regional branches the network received a new, more locally oriented structure.

\subsection{How Sigma is organized}

The management of Sigma regards the company as a virtual organization build up by a network of freelancer consultants and trainers. Trainers and consultants join together in teams in order to realize specific projects. Special positions, e.g. manager of a local branch, require to hold shares of the company. The organization has five business divisions that are managed separately. The boundaries of Sigma are open and fuzzy; newcomers join the organization while others stay only in loose contact or are on their way to leave. Members differ in their level of involvement. While most work full-time for Sigma others do part-time jobs.

A great part of management work is done from an office, located in central Germany; other organizational tasks are distributed, as for example book-keeping which is done by a member in the north of Germany who receives the data via ISDN. Members mostly work from home offices or have a workplace provided by their customer. The eight regional branches are spread over Germany. These branches are orthogonal to business divisions. The number of members of a local branch varies between 10 and 40. Most branches have no office. Local groups meet between biweekly and bimonthly to exchange news, to develop business plans or to give talks about their work practice. Once a year a workshop for all Sigma members is held. Project managers recruit project team members using personal acquaintance or contacts. Due to geographical distribution telephone, fax and computer-based communication are crucial means of communication and collaboration. The bulletin board system SigSys is currently in use by 150 people. SigSys can be operated via modem or ISDN allowing access to discussion groups which deal with regional or projectrelated issues. Furthermore SigSys provides the storage and exchange of binary data in data pools, and e-mail service. The system is regarded as safe compared to the internet and cheaper to operate.

\subsection{Knowledge sharing within Sigma}

In its first years Sigma was a small network of people who knew each other personally. They had come together sharing ideas about collaboration and working practice. At that time telephone, fax and meetings were the means to share information. A few people (e.g. project managers) played a central role in information distribution. Information seekers contacted them to receive desired data. A network of expertise established where everyone knew whom to ask or at least whom to ask to get an expert.

This network was kept during the growth of Sigma. Newcomers made their way into the organization by personal acquaintance with a Sigma member. These guided the new members and served as primary contact points. Almost every interviewee told us that they received a friendly and warm welcome when entering the organization that made them enthusiastic to work and share results. However, the increasing growth accompanied by geographic distribution made it difficult to rely on personal acquaintance for all members.

To continue the principle of personal relationship, regional branches were established that were driven by the 
same goals and ideas as the whole organization. For those whose activities take place within the region this solution is very effective. They regard themselves as part of the branch, belonging to Sigma is less important. People know each other through regular meetings. Most contacts are very close.

During our interviews we observed that within the different branches different cultures and climates have established. While for example group A is very homogeneous group $\mathrm{B}$ shows a greater variety. We tried to identify the reasons for this. The members of branch A stay in close contact by telephone and biweekly meetings. All members belong to a small number of projects within the region though they also work in other projects. The members of branch B belong to several projects with very different background (e.g. information technology vs. human resources). The distribution of new project tasks rises jealousy and competition. Monthly group meetings are attended by subgroups and newcomers. People who work in nation wide projects told us the same. These people regard regional branches as a hindrance to a common identity for Sigma as a whole. Some long-term members think that introducing regional branches has done more harm than good considering a latent competition between the branches and reduced communication between individuals across branches. However, newcomers benefit from local groups and a great part of the members enjoys the opportunity to build contacts on a small scale.

When the management of Sigma perceived a lack of information flow within the organization as a whole they introduced the bulletin board system SigSys. SigSys offers discussion groups; some are open to all members while others are restricted to project teams or regional branches. It took more than a year to achieve a general acceptance of the system. Due to the top-down instead of participatory introduction there was a resistance from IT-people who regarded the system as insufficient compared to other software solutions while non-IT-people had reservations about computer-mediated communication (cf. [24]). SigSys has some technical limitations that restrict information sharing. Within discussion groups no threading is provided which makes it difficult to track a certain topic. The list of discussion members is only provided in a basic manner as a system output. The lack of acknowledge mechanisms makes it hard to use SigSys for timerestricted and formal tasks. For example, a project manager was building a new team and posted an application request in SigSys. When time was running out she was not sure whether her request had been ignored or simply not been read. She had to drop back to the telephone to gather the team members. SigSys' data pools are open to the related group members. Access rights are very coarse grained though it would be important to protect confiden- tial data and to enable a graded control over information [25]. While Sigma members are open to share their data when contacted personally they have great reservations to share anonymously.

Current use of SigSys is a reflection of the opportunities and drawbacks of the system. Its about 150 users access the system from once a week to daily. Most of the topics in open groups deal with organizational matters like announcing meeting dates or deadlines. Some serious discussion topics are spread into this, e.g. perception and improvement of organizational culture. Our interviewees told us that they have a look into SigSys to see what is going on but find it hard to get the important messages out of the rest. Some people who are working part-time within Sigma consider SigSys as a connection line. They usually access SigSys weekly to get an overview about topics and activities although they are aware that only a small part is represented in the system. For a deeper analysis of SigSys cf. [9].

Apart from SigSys internet-based e-mail is used by a lot of members to communicate with project members and customers. The use of computer-mediated communication and collaboration increased during the last two years. In two regional branches where most members are associated to common projects document sharing was introduced and is in heavy use. In project groups e-mail is more common than the use of SigSys. Financial data is processed totally electronic. The book-keeper receives the data via ISDN.

Though the Sigma members know that they have to pull information and know-how. Asked if they push or pull information, apart from some managers, members emphasized the latter. However, as telephone calls are most frequent way of information transfer, providing and requesting information are in fact balanced. This mutual exchange of information and knowledge works twofold: the communication partners receive as much information as they provide and the personal relationship between the partners is enforced. Personal information exchange cannot cover all information that arises within the organization. Who does not know about a topic cannot ask about it. The activities of local branches and topics discussed in working groups are not transparent to all members. Therefore most members criticize the distribution of general information.

\subsection{Measures of improvement}

Members learned to use the different communication means (telephone, fax, SigSys, e-mail) and to select the appropriate one for a task. While this practice is working for every day matters it has not been successful to build up a common knowledge repository. Due to varying use and technical limitations SigSys could not serve as a reliable and effective information distribution means. 
When we joined the "Information and knowledge management" working group it became clear that the work of the group had to be public for all members. We decided to edit a quarterly information letter containing information about the current work of the working group and contributions of Sigma members (e.g. describing their experiences in a project or employing methods of practice). The information letter is available on paper and for download from the SigSys data pool. It is the first regular and official information distribution in the organization beyond personal communication. By making the internal processes of the "Information and knowledge management" group transparent to the Sigma community, a first example of providing information for the whole organization was given.

Lacking transparency affects especially newcomers. They depend heavily on the person who brought them into Sigma. "Ariadne's Thread" (in German "roter Faden"), a web-based prototype, was created to provide a common information source for all members, but with special focus on new members (Figure 2). Like in the ancient myth it shall guide people through the maze of procedures and processes. "Ariadne's Thread" covers information about Sigma's organization and structure and provides space for the presentation of individuals, projects and regional branches. A list of contact persons serves as first aid expertise source for new members and helps to get started with individual inquiries. The members navigate through the web space using a conventional browser. Individuals and project groups edit HTML-pages that are uploaded to the server. Access to the Web space is passwordrestricted.

To prepare the data for "Ariadne's Thread" cooperation of several groups was needed. The work was carried out by members of the "Information and knowledge management" working group. Organizational information was gathered and prepared together with the management. Writing down procedures and regulations, meant to make tacit knowledge explicit. It was the first time that rules and conventions that have developed through the years were discussed. The Web site, though a prototype, serves already as an additional knowledge base for Sigma members. Some project groups as well as individuals and regional groups took the chance to present their activities and profiles on a web side. Especially members who are new to the organization and those not in a central position use the opportunity to promote themselves. Some managers ignore the system. To get them involved into information providing is a challenge.

Motivational factors play a key role in creating necessary incentives for members to provide resources and information to the organization and thus, to form the basis for the development of an information market place. Important in this context are the dynamic personal profiles provided and maintained by Sigma members themselves; they allow each individual to market/promote himself within the organization. Such self-maintained profiles can also help to avoid fundamental problems as known from the discussion on groupware, such as the development of awareness and privacy (cf. $[26,27,28])$. We postulate that decisions about which degree of awareness and privacy is needed have to be made by the user.

By providing information about both the organization and its members, "Ariadne's thread" aims at supporting the distribution of information and know-how. Our provisional observation shows that newcomers and long-term members can benefit from "Ariadne's thread". The new member finds condensed and authentic information about Sigma's organization and procedures. Senior members can retrieve information they have not been aware of while exchanging information through personal contacts, e.g. the activities of a group developing a new business division. Both, juniors and seniors, have the opportunity to build up new work relations based on personal profiles found on the Web site.

After intensive discussions the "Information and knowledge management" working group decided to keep SigSys as the organization wide communication means. We analyzed the system with respect to its capability to support information and knowledge transfer. Besides technical limitations, motivational factors and heterogeneous usage were identified as reasons why the system is less successful than expected. Other aspects like system support and system design (usability) also contribute to the problem. Within the area of usability engineering satisfaction is regarded as an important criterion to increase system use [29]. Since SigSys is the organization wide communication tool we consider it to be important to influence the future development of it. The suggestions concerning the further development include threaded discussion groups and acknowledge mechanisms. Enhanced security and access restriction are of particular importance as the system is not used for the transmission of confidential information as long as access rights cannot be restricted.

While these aspects deal with SigSys' design, we have also been concerned about the process of participatory introduction of the new release. This, however, proves to be difficult due to the geographic distribution and the time pressure of Sigma's members. We are aware that enhancing the technical systems will not be sufficient to change the information and knowledge distribution. Together with the working group on organizational culture, rules and procedures of collaboration are under development that include the usage of technical communication and collaboration means. The major goal is to reestablish an organization wide culture that holds against the diverging forces due to the growth of Sigma. 




Figure 2. "Ariadne's Thread" - Sigma's regional structure (example in English)

\section{Discussion}

As a typical knowledge driven enterprise Sigma is flourishing on behalf of the know-how and the capabilities of its members. While the company was small, information and knowledge could be exchanged through personal communication either by telephone or during meetings. As long as all members knew each others, i.e. they had information about the background, profile and tasks of other people, this form of information sharing and knowledge transfer was sufficient. Members shared common values and goals; they felt a strong cohesion. A trustful environment made successful collaboration easy.

The rapid growth in members required organizational restructuring. By establishing regional branches Sigma tried to preserve its communication culture. This strategy was partly successful. As shown by our interviews people in local branches stay in close personal contact and keep each other up to date by regular meetings and informal telephone conversations. However, not all members feel themselves belonging to a local branch. These people depend on the availability of organization wide information distribution

Information and knowledge flow was strictly bound to personal relationship. In the growing enterprise knowledge about internal topics was no longer distributed homogeneously. Instead, knowledge islands emerged, coupled through personal contacts. Those belonging to such an island felt well-informed while others, already distant, were losing connection. The regional branches began to develop local culture and 
drifted away from the company's global processes. A general transparency was missing. Organization wide communication and information means were needed.

Continuing Sigma's highly communicative tradition, the management decided to introduce a bulletin board system. Theoretically, SigSys is suitable for information and knowledge transfer. Discussion groups enable people to share information and exchange opinions. Though geographically distributed, Sigma members can stay in touch and follow activities in other groups and the management. What was not foreseen is the fact that the communication style provided by the bulletin board system SigSys differs from the former tradition within Sigma. While personal information exchange is always balanced, consisting of mutual contributions, the bulletin board allows to gather anonymously information without putting anything in. Individual access differs in frequency and intensity, some members do not use it at all. Since no usage conventions concerning SigSys were established, the system could not serve as a reliable communication means for all members. Moreover, SigSys competes with other computer-based communication means like internet-based e-mail, especially in project work and confidential communication.

According to Media Richness Theory [30] a communication medium can be ranked on its ability to handle equivocality and uncertainty. In practice, situation requirements matched against media characteristics as well as social and organizational factors influence media choice $[31,32]$, among these are message urgency and content length. The size of the user base is considered as critical for a general system acceptance [33].

Our observation of communication media use within Sigma suggests to distinguish between one-to-one and group communication. Further, communication means differ in their capability to balance pushing and pulling of information. A combination of unbalanced information flow and group communication can be considered as critical. This combination requires particular attention in system design and usage regulations.

The prototype "Ariadne's Thread" has been designed as a knowledge base for the whole organization where all members can pull information. By making activities transparent and inviting Sigma members to participate, the involvement of the whole organization is initiated. The need to write down organizational information leads to externalizing regulations, conventions and procedures which were tacit knowledge before. Developing these contents can bring diverging groups back to common ground and reinforce relationships as they are involved in a common process. However, as the individual members work under time pressure and with subjective goals it can be difficult to include them in this process. This is a drawback of the lightweight structure of virtual organi- zations that does not provide organizational resources for general services [9].

From the beginning it was clear to all participants that "Ariadne's Thread" serves as an information base open to all members. While the bulletin board is a group communication means, the Web site serves as an information reference point so far not present in the virtual organization Sigma. Voluntary work is motivated by the opportunity to promote oneself. The presentation of groups and members is managed by the users themselves ensuring control and responsibility.

Cohesion within the organization needed revitalization. The values and ideas of the foundation are still alive within Sigma though partially weakened and shifted by growth, regional branches and geographical distribution. On the personal level knowledge and information exchange are working based on mutual trust. This local climate is the culture of Sigma's foundation. The challenge is to transfer it to the organizational level and to adapt it to the structural change Sigma underwent during the last years. The work on establishing a webbased knowledge base contributes to the effort to develop a new organizational culture. It initiated a process of externalizing procedures and regulations. All members involved in this process are creating the organization's culture.

The usage of SigSys and "Ariadne's Thread" is a good example of the interplay between technology and organization $[34,35]$. Without usage regulations heterogeneous practices evolve that prevent to benefit from the common infrastructure. General conventions for the usage of both systems (SigSys and "Ariadne's Thread") are under development. These rules will be embedded into a set of statements that define Sigma's information and knowledge policy. While SigSys serves for every day group communication the Web site serves as an information repository. We expect that the redesign of SigSys together with an appropriate usage guide will enhance the suitability of the system. A change in usage should follow, supported by an accommodation phase when people are guided in the use of the redesigned SigSys.

To build flexible teams is one of the enabling factors of virtual organizations. Two factors are essential to support the establishment of teams. First, providing information about skills and experience helps to overcome problems arising from the absence of personal relationships. The use of "Ariadne's Thread" as a market place to promote oneself is an example for this. The information is provided by the particular owner avoiding privacy concerns. Second, the procedure of establishing a team is often done under time pressure. The team manager needs support mechanisms that provide feedback as to whether a person is interested, not interested, not available, does not feel competent, etc. Neither the 
Web site nor SigSys offer appropriate tools. Shared schedules of appointments, including predefined answer formulas, seem to be useful in that context [36].

The example of Sigma demonstrated some mechanisms of knowledge sharing. A living tradition of oral and personal information distribution cannot simply be replaced by introducing computer-mediated communication and information systems. As these technical systems provide a more anonymous access, mutual information exchange is not granted. Technical systems are useful when other options are not available, e.g. to keep "in touch" without expenditure or to provide information to the whole community. Knowledge transfer takes place in two ways: Active transfer requires a bidirectional channel, ensuring the flow of information between the participants. Passive transfer is an offer to pull information without favour in return. Therefore, the information provider will only take the costs when some benefit is expected. Within the fluid virtual organization passive knowledge transfer is vital to provide a reliable information reference point.

\section{Conclusion and future work}

Technical information and communication systems, embedded into a strong organizational culture that emphasizes the importance of knowledge transfer are the skeleton of a fluid virtual organization. To achieve sustained knowledge management all members and levels of the organization must cooperate. This becomes possible when everyone is participating in a process of developing common goals, values and procedures. Knowledge sharing requires mutual trust. By providing transparency about ongoing activities and openness for participation from all members a trustful environment is created.

As information and knowledge always flow through several channels consisting of different technologies and communication media, knowledge management means to guide the usage of these channels according to conventions to achieve greater coherence. By embedding knowledge management in the organizational culture, procedures and values must be developed that foster information and knowledge sharing. Knowledge management is an evolutionary process that needs embedding into organizational culture.

The introduction of new communication or information technologies that are capable of enhancing knowledge sharing can be used to initiate a knowledge culture. The introduction process initializes the externalization of tacit knowledge and reflection on established information flows. Building up a permanent organizational memory means to include all members of the organization in its construction. The participatory development of contents, rules and goals creates cohesion forces and becomes part of the organizational culture provided that the necessary organizational are available.

Within Sigma the prototype of an organization wide information base has been established. The next step will be to extend its functions to support team-related requirements like team building and project documentation. When the new version of SigSys will be introduced we will study the interplay of usage rules and enhanced functionality.

\section{Acknowledgement}

Special thanks to Volkmar Pipek for valuable hints and discussions, and to the anonymous reviewers for their suggestions.

\section{References}

[1] Nonaka I. and Takeushi H., The Knowledge-Creating Company, Oxford University Press, New York, 1995.

[2] Davenport Th. H. and Prusak L., Working Knowledge: How Organizations Manage What They Know, Harvard Business School Press, Boston, MA, 1998.

[3] Davidow H. W. and Malone M.S., The Virtual Corporation, Structuring and Revitalizing the Corporation for the 21st Century, Harper Collins, New York, 1992.

[4] Strausak N., "Resumée of VoTalk", in Sieber, P.; Griese, J. (Eds.) Organizational Virtualness, Proceedings of the VoNet Workshop, April 27-28, Bern, 1998, pp. 9-24.

[5] Warnecke H.-J., Revolution der Unternehmenskultur, Springer, Berlin, 1993.

[6] Welles E., "Virtual Realities", Inc Cover Story, Inc., vol 14, 8, 1993, pp. 50-58.

[7] Miller D.B., Clemons E.K. and Row M.C., "Information Technology and the Global Virtual Corporation", in S.P. Bradley, J.A. Hutchinson, \& R.L. Nolan (eds.), Globalization, Technology and Competition, Boston: Harvard Business School Press, 1993, pp. 283-307.

[8] Mowshowitz A., "Virtual Organization", Communications of the ACM, Vol 40, No. 9, 1997, pp. 30-37.

[9] Rittenbruch M., Kahler H., and Cremers A. B., "Supporting Cooperation in a Virtual Organization", 19 1 th Annual International Conference on Information Systems, Helsinki, Finland, 1998, pp. 30-38.

[10] Nohria N., and Berkley J.D., "The Virtual Organization: Bureaucracy, Technology, and the Implosion of Control", in C. Hekscher and A. Donnellon (eds.), The Post-Bureaucratic Organization: New Perspectives in Organizational Change, Sage, Thousand Oaks, California, 1994, pp. 108-128.

[11] Jarvenpaa S. and Shaw T.R., "Global Virtual Teams: Integrating Models of Trust", in Sieber P.; Griese J. (eds): Organizational Virtualness, Proceedings of the VoNet Workshop 1998, April 27-28, Bern, Simowa-Verlag, 1998. 
[12] Ellis C.A., Gibbs S.J. and Rein G.L., "Groupware: Overview and Perspectives. Some issues and experiences", Communication of the ACM, (34:1), 1991, pp. 18-29.

[13] Krebs M., "Die virtuelle Unternehmung als Wissensorganisation: Potentiale und Grenzen des Wissensmanagements", Arbeitspapiere des FB Wirtschaftwissenschaften, Universität Wuppertal, Nr. 181, 1998.

[14] Lipnack J. and Stamps J., Virtual Teams, John Wiley \& Sons, 1997.

[15] Hofstede G., Neuijen B., Ohayu, D. D. and Sanders, G., "Measuring organizational cultures: A qualitative and quantitative study across twenty cases", Administrative Science Quarterly, 35, 1990, pp. 286-316.

[16] Schein E. H., "What is culture", in P. J. Frost, L. F. Moore, M. R. Louis, C. C. Lundberg, \& J. Martin (Eds.), Reframing organizational culture, Newbury Park, CA: Sage, 1991, pp. 243-253.

[17] Kahler H. and Rohde M., "Changing to stay itself", SIGOIS Bulletin, Vol. 17, No. 3, 1996, pp. 62-64.

[18] Lindstaedt St. N. and Schneider K., "Bridging the Gap between Face-to-Face Communication and Long-Term Collaboration", GROUP97, 1997, pp. 331-340.

[19] Stein E. and Zwass V., "Actualizing Organizational Memory with Information Systems", Information System Research, Vol 6, No. 2, 1995, pp. 85-117.

[20] Walsh J. and Ungson G., "Organizational Memory", Academy of Management Review, Vol 16, No. 1, 1991, pp. 5791.

[21] Ackerman M. S., "Augmenting the Organizational Memory: A Fiel Study of Answer Garden", Proceedings of the Conference on Computer Supported Cooperative Work, Chapel Hill, NC, USA, 1994, pp. 243-252.

[22] Bannon L. and Kuutti K., "Shifting Perspectives on Organizational Memory: From Storage to Active Remembering", in: Nunamaker J. F., Sprague R. H. (eds): Proceedings of the 29th Hawaii International Conference on System Sciences, Vol. 3, 1996, pp. 156-167.

[23] McDonald D.W. and Ackerman M., "Just Talk To Me: A Field Study of Expertise Location", Proc. Conference on Computer-Supported Cooperative Work (CSCW'98), Seattle, 14.-18.11.1998, ACM Press, New York, 1998.[24] Namioka A. and Schuler D., Participatory Design: Principles and Practices, Lawrence Earlbaum, 1993.

[25] Shen H. and Dewan P., "Access Control for Collaborative Environments", Proceedings of the CSCW'92, Toronto, ACM Press, New York, 1992, pp. 51-58.

[26] McDaniel S.E. and Brinck T., "Awareness in Collaborative Systems", SIGCHI Bulletin (29:4), 1997, pp. 68-71.

[27] Bellotti V. and Sellen, A., "Design for Privacy in Ubiquitous Computing Enviroments", Proceedings of the third European Conference on Computer-Supported Cooperative Work, Milan, Italy, September 13.-17., 1993, pp. 77-92.
[28] Clement A., "Considering Privacy in the Development of Multi-Media Communication", Proceedings of the CSCW '94, NC, USA, University of Toronto, 1994, pp. 67-88.

[29] Nielsen J., Usability Engineering, AP Professional, Boston, 1994.

[30] Daft R. L. and Lengel R. J., "Information Richness: A new Approach to Managerial Behaviour and Organization Design", in Staw B. M., Cummings L.L. (eds), Research in Organizational Behaviour, JAI Press, Greenwich, 1984, pp. 191-233.

[31] Caldwell B. S., Uang Sh.-T. and Taha L. H., "Appropriateness of communications media use in organizations: situation requirements and media characteristics", Behaviour and Information Technology, Vol 14, No. 4, 1995, pp. 199207.

[32] El-Shinnawy M. and Markus M. L., " The poverty of media richness theory: explaining people's choice of electronic mail vs. voice mail", Int. J. Human-Computer Studies, 46, 1997, pp. 443-467.

[33] Grudin J., "Groupware and social dynamics: Eight challenges for developers", Communications of the ACM (37:1), 1994, pp. 93-105.

[34] Stiemerling O. and Wulf V., Rohde M., "Integrated Organization and Technology Development - The case of the ORGTechProject", Proceedings of Concurrent Engineering (CE 98), Tokio, July 13 - 15, 1998.

[35] Orlikowski W., "Learning from Notes: Organizational Issues in Groupware Implementation", Proceedings of the CSCW' '92, 1992, pp. 362-369.

[36] Grudin J. and Palen, L., "Why groupware succeeds: Discretion or mandate?", Proceedings of the ECSCW'95, Dordrecht, The Netherlands, Kluwer, 1995, pp. 263-278. 